\title{
Qualitative Behavior of Rational Difference Equation of Big Order
}

\author{
M. M. El-Dessoky ${ }^{1,2}$ \\ ${ }^{1}$ Mathematics Department, Faculty of Science, King Abdulaziz University, P.O. Box 80203, Jeddah 21589, Saudi Arabia \\ ${ }^{2}$ Mathematics Department, Faculty of Science, Mansoura University, Mansoura 35516, Egypt
}

Correspondence should be addressed to M. M. El-Dessoky; dessokym@mans.edu.eg

Received 4 February 2013; Accepted 20 April 2013

Academic Editor: Cengiz Çinar

Copyright (c) 2013 M. M. El-Dessoky. This is an open access article distributed under the Creative Commons Attribution License, which permits unrestricted use, distribution, and reproduction in any medium, provided the original work is properly cited.

We investigate the global convergence, boundedness, and periodicity of solutions of the recursive sequence $x_{n+1}=\left(a x_{n-l}+\right.$ $\left.b x_{n-x}\right) /\left(c+d x_{n-l} x_{n-k}\right), n=0,1, \ldots$, where the parameters $a, b, c$, and $d$ are positive real numbers, and the initial conditions $x_{-t}, x_{-t+1}, \ldots, x_{-1}$ and $x_{0}$ are positive real numbers where $t=\max \{k, l\}$.

\section{Introduction}

Recently, there has been a lot of interest in studying the global attractivity, the boundedness character, and the periodicity nature of nonlinear difference equations see for example, [122].

The study of the nonlinear rational difference equations of a higher order is quite challenging and rewarding, and the results about these equations offer prototypes towards the development of the basic theory of the global behavior of nonlinear difference equations of a big order; recently, many researchers have investigated the behavior of the solution of difference equations. For example, in [8]. Elabbasy et al. investigated the global stability and periodicity character and gave the solution of special case of the following recursive sequence:

$$
x_{n+1}=a x_{n}-\frac{b x_{n}}{c x_{n}-d x_{n-1}} \text {. }
$$

Elabbasy et al. [9] investigated the global stability, boundedness, and periodicity character and gave the solution of some special cases of the difference equation

$$
x_{n+1}=\frac{\alpha x_{n-k}}{\beta+\gamma \prod_{i=0}^{k} x_{n-i}} .
$$

Elabbasy et al. [10] investigated the global stability and periodicity character and gave the solution of some special cases of the difference equation

$$
x_{n+1}=\frac{d x_{n-l} x_{n-k}}{c x_{n-s}-b}+a .
$$

Saleh and Aloqeili [23] investigated the difference equation

$$
y_{n+1}=A+\frac{y_{n}}{y_{n-k}}, \quad \text { with } A<0 .
$$

Wang et al. [24] studied the global attractivity of the equilibrium point and the asymptotic behavior of the solutions of the difference equation

$$
x_{n+1}=\frac{a x_{n-l} x_{n-k}}{\alpha+b x_{n-s}+c x_{n-t}} .
$$

In [25], Wang et al. investigated the asymptotic behavior of equilibrium point for a family of rational difference equation

$$
x_{n+1}=\frac{\sum_{i=1}^{t} A_{s_{i}} x_{n-s_{i}}}{B+C \prod_{j=1}^{k} x_{n-t_{j}}}+D x_{n} .
$$

Yalçinkaya [26] considered the dynamics of the difference equation

$$
x_{n+1}=\alpha+\frac{x_{n-m}}{x_{n}^{k}} .
$$


Zayed and El-Moneam [27, 28] studied the behavior of the following rational recursive sequences:

$$
x_{n+1}=a x_{n}-\frac{b x_{n}}{c x_{n}-d x_{n-k}}, \quad x_{n+1}=\frac{\alpha+\beta x_{n}+\gamma x_{n-1}}{A+B x_{n}+C x_{n-1}} .
$$

For some related works see [29-39].

Our goal in this paper is to investigate the global stability character and the periodicity of solutions of the recursive sequence

$$
x_{n+1}=\frac{a x_{n-l}+b x_{n-k}}{c+d x_{n-l} x_{n-k}}, \quad n=0,1, \ldots,
$$

where the parameters $a, b, c$, and $d$ are positive real numbers and the initial conditions $x_{-t}, x_{-t+1}, \ldots, x_{-1}$ and $x_{0}$ are positive real numbers where $t=\max \{k, l\}$.

\section{Local Stability of the Equilibrium Point of (9)}

This section deals with the local stability character of the equilibrium point of (9)

Equation (9) has equilibrium points given by

$$
\bar{x}=\frac{(a+b) \bar{x}}{c+d \bar{x}^{2}}
$$

then

$$
\bar{x}\left\{d \bar{x}^{2}+c-a-b\right\}=0 .
$$

Then the equilibrium points of (9) are given by

$$
\bar{x}=0 \quad \text { or } \quad \bar{x}=\sqrt{\frac{a+b-c}{d}} \text { when } a+b>c .
$$

Let $f:(0, \infty)^{2} \rightarrow(0, \infty)$ be a continuously differentiable function defined by

$$
f(u, v)=\frac{a u+b v}{c+d u v}
$$

Therefore, it follows that

$$
\frac{\partial f(u, v)}{\partial u}=\frac{a c-b d v^{2}}{(c+d u v)^{2}}, \quad \frac{\partial f(u, v)}{\partial v}=\frac{b c-a d u^{2}}{(c+d u v)^{2}} .
$$

Theorem 1. The following statements are true.

(1) If $a+b \leq c$, then the only equilibrium point $\bar{x}=0$ of (9) is locally stable.

(2) If $a+b>c$, then the positive equilibrium point $\bar{x}=$ $\sqrt{(a+b-c) / d}$ of (9) is locally stable if $|c-b|+|c-a|<$ $a+b$.

Proof. (1) If $a+b \leq c$, then we see from (14) that

$$
\frac{\partial f(0,0)}{\partial x_{n-l}}=\frac{a}{c}, \quad \frac{\partial f(0,0)}{\partial x_{n-k}}=\frac{b}{c} .
$$

Then, the linearized equation associated with (9) about $\bar{x}=0$ is

$$
y_{n+1}-\frac{a}{c} y_{n-l}-\frac{b}{c} y_{n-k}=0,
$$

whose characteristic equation is

$$
\lambda^{k+1}-\frac{a}{c} \lambda^{k-l}-\frac{b}{c}=0 .
$$

Then, (16) is asymptotically stable if $a+b<c$, and then the equilibrium point $\bar{x}=0$ of (9) is locally stable.

(2) If $a+b>c$, then we see from (14) that

$$
\begin{aligned}
& \frac{\partial f(\bar{x}, \bar{x})}{\partial x_{n-l}}=\frac{a c-b d((a+b-c) / d)}{(c+d((a+b-c) / d))^{2}}=\frac{c-b}{a+b}, \\
& \frac{\partial f(\bar{x}, \bar{x})}{\partial x_{n-k}}=\frac{b c-a d((a+b-c) / d)}{(c+d((a+b-c) / d))^{2}}=\frac{c-a}{a+b} .
\end{aligned}
$$

Then, the linearized equation of (9) about $\bar{x}$ is

$$
y_{n+1}-\frac{c-b}{a+b} y_{n-l}-\frac{c-a}{a+b} y_{n-k}=0
$$

whose characteristic equation is

$$
\lambda^{k+1}-\frac{c-b}{a+b} \lambda^{k-l}-\frac{c-a}{a+b}=0 .
$$

Then, (19) is asymptotically stable if all roots of (20) lie in the open $\operatorname{disc}|\lambda|<1$, that is, if

$$
\left|\frac{c-b}{a+b}\right|+\left|\frac{c-a}{a+b}\right|<1
$$

which is true if

$$
|c-b|+|c-a|<a+b .
$$

The proof is complete.

\section{Boundedness of the Solutions of (9)}

Here, we study the boundedness nature of the solutions of (9).

Theorem 2. Every solution of (9) is bounded if $c>a+b$.

Proof. Let $\left\{x_{n}\right\}_{n=-t}^{\infty}$ be a solution of (9). It follows from (9) that

$$
x_{n+1}=\frac{a x_{n-l}+b x_{n-k}}{c+d x_{n-l} x_{n-k}} \leq \frac{a x_{n-l}+b x_{n-k}}{c} .
$$

By using a comparison, we can write the right-hand side as follows:

$$
y_{n+1}=\frac{a y_{n-l}}{c}+\frac{b y_{n-k}}{c},
$$

and this equation is locally asymptotically stable if $a+b<c$ and converges to the equilibrium point $\bar{y}=0$.

Therefore,

$$
\limsup _{n \rightarrow \infty} x_{n}=0 \text {. }
$$

Thus, the solution is bounded. 


\section{Existence of Periodic Solutions}

In this section, we study the existence of periodic solutions of (9). The following theorem states the necessary and sufficient conditions that this equation has periodic solutions of prime period two.

Theorem 3. Equation (9) has a prime period two solutions if and only if one of the following statements holds:

(1) $a+b-c>0$, and $l, k-o d d$,

(2) $a+c-b>0$, and $k$-odd, l-even,

(3) $b+c-a>0$, and $l$-odd, $k$-even.

Proof. We will prove the theorem when condition (1) is true, and the proof of the other cases is similar and so we will be omit it.

First suppose that there exists a prime period two solution

$$
\ldots, p, q, p, q, \ldots \text {, }
$$

of (9). We will prove that Condition (1) holds.

We see from (9) that

$$
p=\frac{(a+b) p}{c+d p^{2}}, \quad q=\frac{(a+b) q}{c+d q^{2}} .
$$

Then,

$$
\begin{aligned}
& c+d p^{2}=a+b, \\
& c+d q^{2}=a+b .
\end{aligned}
$$

Subtracting (28) from (29) gives

$$
d\left(p^{2}-q^{2}\right)=0
$$

Since $p \neq q$, it follows that

$$
p=-q
$$

Again, from (28) and (29)

$$
p^{2}=q^{2}=\frac{a+b-c}{d},
$$

and so

$$
a+b-c>0 \text {. }
$$

Therefore, inequality (1) holds.

Second, suppose that inequality (1) is true. We will show that (9) has a prime period two solution.

Assume that

$$
p=+\sqrt{\frac{a+b-c}{d}}, \quad q=-\sqrt{\frac{a+b-c}{d}} .
$$

We see from inequality (1) that

$$
a+b-c>0 \text {. }
$$

Therefore, $p$ and $q$ are distinct real numbers.
Set

$$
x_{-l}=x_{-k}=p, \quad x_{-2}=q, \quad x_{-1}=p, \quad x_{0}=q .
$$

We wish to show that

$$
x_{1}=x_{-1}=p, \quad x_{2}=x_{0}=q .
$$

It follows from (9) that

$$
x_{1}=\frac{(a+b) p}{c+d p^{2}}=\frac{(a+b) \sqrt{(a+b-c) / d}}{c+d((a+b-c) / d)}=\sqrt{\frac{a+b-c}{d}}=p .
$$

Similarly, we see that

$$
\begin{aligned}
x_{2} & =\frac{(a+b) q}{c+d q^{2}}=\frac{-(a+b) \sqrt{(a+b-c) / d}}{c+d((a+b-c) / d)} \\
& =-\sqrt{\frac{a+b-c}{d}}=q .
\end{aligned}
$$

Then, it follows by induction that

$$
x_{2 n}=q, \quad x_{2 n+1}=p, \quad \forall n \geq-1 .
$$

Thus, (9) has the prime period two solution

$$
\ldots, p, q, p, q, \ldots,
$$

where $p$ and $q$ are distinct roots of a quadratic equation, and the proof is complete.

\section{Global Attractor of the Equilibrium Point of (9)}

In this section, we investigate the global asymptotic stability of (9). If we take the function $f(u, v)$ defined by (16), then we have four cases of the monotonicity behavior in its arguments (all of these cases we suppose that $a+b>c$ ).

Theorem 4. If the function $f(u, v)$ defined by (16) is nondecreasing (or nonincreasing) in $u, v$, then the positive equilibrium point $\bar{x}=\sqrt{(a+b-c) / d}$ is a global attractor of (9).

Proof. Let $\left\{x_{n}\right\}_{n=-t}^{\infty}$ be a solution of (9) and again let $f$ be a function defined by (16).

We will prove the theorem when $f(u, v)$ is nondecreasing and the proof of the other cases is similar, and so we will omit it.

Suppose that $(m, M)$ is a solution of the systems $M=$ $f(M, M)$ and $m=g(m, m)$. Then, from (9), we see that

$$
M=\frac{a M+b M}{c+d M^{2}}, \quad m=\frac{a m+b m}{c+d m^{2}},
$$

or

$$
c+d M^{2}=a+b, \quad c+d m^{2}=a+b .
$$


Subtracting these two equations, we obtain

$$
d(M-m)(M+m)=0 .
$$

Under the condition $d>0$, we see that

$$
M=m \text {. }
$$

It follows by Theorem 2 that $\bar{x}$ is a global attractor of (9), and then the proof is complete.

Theorem 5. If the function $f(u, v)$ defined by (16) is nondecreasing in $u$ and nonincreasing in $v$, then the positive equilibrium point $\bar{x}=\sqrt{(a+b-c) / d}$ is a global attractor of (9) if $c+b>a$.

Proof. Let $\left\{x_{n}\right\}_{n=-t}^{\infty}$ be a solution of (9) and again let $f$ be a function defined by (16).

Suppose that $(m, M)$ is a solution of the systems $M=$ $f(M, m)$ and $m=g(m, M)$. Then, from (9), we see that

$$
M=\frac{a M+b m}{c+d m M}, \quad m=\frac{a m+b M}{c+d m M},
$$

or

$$
\begin{aligned}
& c M+d m M^{2}=a M+b m, \\
& c m+d M m^{2}=a m+b M .
\end{aligned}
$$

Subtracting these two equations, we obtain

$$
\begin{gathered}
c(M-m)+d M m(M-m)=(a-b)(M-m), \\
(M-m)\{c+b-a+d M m\}=0 .
\end{gathered}
$$

Under the condition $c+b>a$, we see that

$$
M=m \text {. }
$$

It follows by Theorem 2 that $\bar{x}$ is a global attractor of (9), and then the proof is complete.

Theorem 6. If the function $f(u, v)$ defined by (16) is nondecreasing in $v$, nonincreasing in $u$. Then the positive equilibrium point $\bar{x}=\sqrt{(a+b-c) / d}$ is a global attractor of (9) if $c+a>b$.

Proof. The proof is similar to the previous Theorem and so we will be omit it.

Lemma 7. When $c \geq a+b$ then the equilibrium point $\bar{x}=0$ of (9) is global attractor.

Proof. If $c \geq a+b$, then the proof follows by Theorem 2 .

\section{Numerical Examples}

For confirming the results of this paper, we consider numerical examples which represent different types of solutions to (9).

Example 1. We assume that $l=1, k=2, x_{-2}=3, x_{-1}=$ $2, x_{0}=6, a=2, b=5, c=8$, and $d=6$. See Figure 1 .



FIGURE 1: It shows the solution of (9) with $l=1, k=2, x_{-2}=$ $3, x_{-1}=2, x_{0}=6, a=2, b=5, c=8$, and $d=6$.



FIGURE 2: It shows the behavior of the solution of (9) with $l=1, k=$ $3, x_{-3}=3, x_{-2}=1, x_{-1}=6, x_{0}=5, a=9, b=13, c=$ 0.1 , and $d=2$.

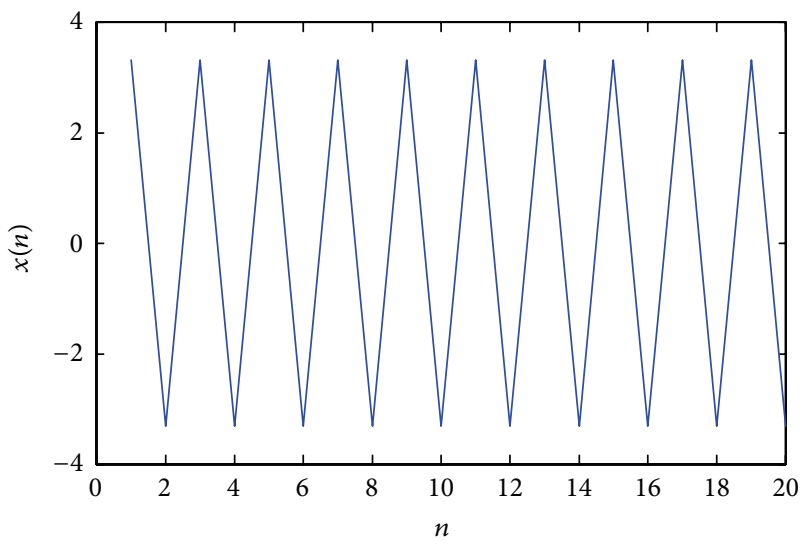

FIGURE 3: It shows the periodicity of the solution of (9) when $l=$ $3, k=1, x_{-3}=x_{-1}=p, x_{-2}=x_{0}=q, a=9, b=13, c=$ 0.1 , and $d=2$. 


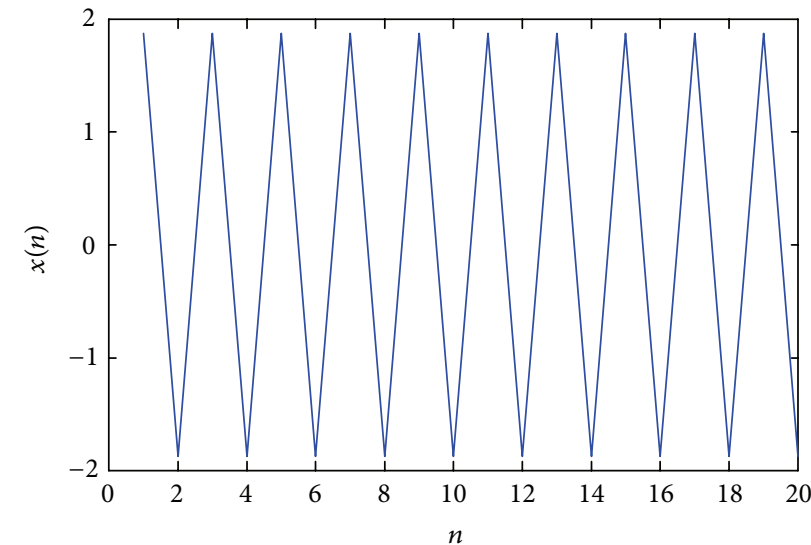

FIGURE 4: It shows the periodicity of the solution of (9) when $l=$ $4, k=3, x_{-3}=x_{-1}=q, x_{-4}=x_{-2}=x_{0}=p, a=9, b=5, c=$ 3 , and $d=2$.

Example 2. See Figure 2, since $l=1, k=3, x_{-3}=3, x_{-2}=$ $1, x_{-1}=6, x_{0}=5, a=9, b=13, c=0.1, d=2$.

Example 3. Figure 3 shows the solutions when $l=3, k=$ $1, x_{-3}=x_{-1}=p, x_{-2}=x_{0}=q, a=9, b=13, c=$ 0.1 , and $d=2$. (Since $p, q= \pm \sqrt{(a+b-c) / d}$ ).

Example 4. Figure 4 shows the solutions when $l=4, k=$ $3, x_{-3}=x_{-1}=q, x_{-4}=x_{-2}=x_{0}=p, a=9, b=5, c=$ 3 , and $d=2$. (Since $p, q= \pm \sqrt{(a+b-c) / d}$ ).

\section{Acknowledgments}

This work was funded by the Deanship of Scientific Research (DSR), King Abdulaziz University, Jeddah, under Grant no. (130-056-D1433). The author, therefore, acknowledges with thanks to DSR technical and financial support.

\section{References}

[1] R. Abu-Saris, C. Çinar, and I. Yalçinkaya, "On the asymptotic stability of $x_{n+1}=a+x_{n} x_{n-k} /\left(x_{n}+x_{n-k}\right)$," Computers \& Mathematics with Applications, vol. 56, no. 5, pp. 1172-1175, 2008.

[2] R. P. Agarwal, Difference Equations and Inequalities, Marcel Dekker, New York, NY, USA, 1st edition, 1992.

[3] R. P. Agarwal, Difference Equations and Inequalities, Marcel Dekker, New York, NY, USA, 2nd edition, 2000.

[4] R. P. Agarwal and E. M. Elsayed, "Periodicity and stability of solutions of higher order rational difference equation," $A d-$ vanced Studies in Contemporary Mathematics, vol. 17, no. 2, pp. 181-201, 2008.

[5] M. Aloqeili, "Dynamics of a rational difference equation," Applied Mathematics and Computation, vol. 176, no. 2, pp. 768774, 2006.

[6] N. Battaloglu, C. Cinar, and I. Yalçınkaya, "The dynamics of the difference equation," Ars Combinatoria, vol. 97, pp. 281-288, 2010 .
[7] C. Çinar, "On the positive solutions of the difference equation $x_{n+1}=a x_{n-1} /\left(1+b x_{n} x_{n-1}\right)$," Applied Mathematics and Computation, vol. 156, no. 2, pp. 587-590, 2004.

[8] E. M. Elabbasy, H. El-Metwally, and E. M. Elsayed, "On the difference equation $x_{n+1}=a x_{n}-\left(b x_{n} / c x_{n}-d x_{n-1}\right)$," Advances in Difference Equations, vol. 2006, Article ID 82579, 10 pages, 2006.

[9] E. M. Elabbasy, H. El-Metwally, and E. M. Elsayed, "On the difference equation $x_{n+1}=a x_{n-k} /\left(\beta+\gamma \prod_{i=0}^{k} x_{n-i}\right)$," Journal of Concrete and Applicable Mathematics, vol. 5, no. 2, pp. 101-113, 2007.

[10] E. M. Elabbasy, H. El-Metwally, and E. M. Elsayed, "Qualitative behavior of higher order difference equation," Soochow Journal of Mathematics, vol. 33, no. 4, pp. 861-873, 2007.

[11] S. Kang and B. Shi, "Periodic solutions for a system of difference equations," Discrete Dynamics in Nature and Society, vol. 2009, Article ID 760328, 9 pages, 2009.

[12] A. Y. Özban, "On the system of rational difference equations $x_{n}=a / y_{n-3}, y_{n}=b y_{n-3} / x_{n-q} y_{n-q}$, Applied Mathematics and Computation, vol. 188, no. 1, pp. 833-837, 2007.

[13] E. Camouzis and G. Papaschinopoulos, "Global asymptotic behavior of positive solutions on the system of rational difference equations $x_{n+1}=1+x_{n} / y_{n-m}, y_{n+1}=1+y_{n} / x_{n-m}$," Applied Mathematics Letters, vol. 17, no. 6, pp. 733-737, 2004.

[14] E. M. Elabbasy and E. M. Elsayed, "Global asymptotic behavior attractivity and periodic nature of a difference equation," World Applied Sciences Journal, vol. 12, no. 1, pp. 39-47, 2011.

[15] V. L. Kocić and G. Ladas, Global Behavior of Nonlinear Difference Equations of Higher Order with Applications, Kluwer Academic, Dordrecht, The Netherlands, 1993.

[16] M. R. S. Kulenović and G. Ladas, Dynamics of Second Order Rational Difference Equations with Open Problems and Conjectures, Chapman \& Hall/CRC, Boca Raton, Fla, USA, 2001.

[17] I. Yalçinkaya, "On the global asymptotic stability of a secondorder system of difference equations," Discrete Dynamics in Nature and Society, vol. 2008, Article ID 860152, 12 pages, 2008.

[18] H. El-Metwally, "Global behavior of an economic model," Chaos, Solitons \& Fractals, vol. 33, no. 3, pp. 994-1005, 2007.

[19] I. Yalçinkaya, "On the global asymptotic behavior of a system of two nonlinear difference equations," Ars Combinatoria, vol. 95, pp. 151-159, 2010.

[20] I. Yalçinkaya, C. Çinar, and M. Atalay, "On the solutions of systems of difference equations," Advances in Difference Equations, vol. 2008, Article ID 143943, 9 pages, 2008.

[21] J. Diblík, B. Iricanin, S. Stevic, and Z. Šmarda, "On some symmetric systems of difference equations," Abstract and Applied Analysis, vol. 2013, Article ID 246723, 7 pages, 2013.

[22] E. M. Elsayed, "Dynamics of a recursive sequence of higher order," Communications on Applied Nonlinear Analysis, vol. 16, no. 2, pp. 37-50, 2009.

[23] M. Saleh and M. Aloqeili, "On the difference equation $y_{n+1}=$ $A+\left(y_{n} / y_{n-k}\right)$ with $A<0$," Applied Mathematics and Computation, vol. 176, no. 1, pp. 359-363, 2006.

[24] C. Wang, S. Wang, Z. Wang, H. Gong, and R. Wang, "Asymptotic stability for a class of nonlinear difference equation," Discrete Dynamics in Natural and Society, vol. 2010, Article ID 791610, 10 pages, 2010.

[25] C.-y. Wang, Q.-h. Shi, and S. Wang, "Asymptotic behavior of equilibrium point for a family of rational difference equations," Advances in Difference Equations, vol. 2010, Article ID 505906, 10 pages, 2010. 
[26] I. Yalçinkaya, "On the difference equation $x_{n+1}=\alpha+\left(x_{n-m} / x_{n}^{k}\right)$," Discrete Dynamics in Nature and Society, vol. 2008, Article ID 805460, 8 pages, 2008.

[27] E. M. E. Zayed and M. A. El-Moneam, "On the rational recursive sequence," Communications on Applied Nonlinear Analysis, vol. 15, no. 2, pp. 47-57, 2008.

[28] E. M. E. Zayed and M. A. EL-Moneam, "On the rational recursive sequence $x_{n+1}=\alpha+\beta x_{n}+\gamma x_{n-1} /\left(A+B x_{n}+C x_{n-1}\right)$," Communications on Applied Nonlinear Analysis, vol. 12, no. 4, pp. 15-28, 2005.

[29] E. M. Elsayed and M. M. El-Dessoky, "Dynamics and behavior of a higher order rational recursive sequence," Advances in Difference Equations, pp. 2012-69, 2012.

[30] D. Simsek, B. Demir, and C. Cinar, "On the solutions of the system of difference equations $x_{n+1}=\max \left\{A / x_{n}, y_{n} / x_{n}\right\}, y_{n+1}=$ $\max \left\{A / y_{n}, x_{n} / y_{n}\right\}, "$ Discrete Dynamics in Nature and Society, vol. 2011, Article ID 325296, 11 pages, 2009.

[31] M. Mansour, M. M. El-Dessoky, and E. M. Elsayed, “The form of the solutions and periodicity of some systems of difference equations," Discrete Dynamics in Nature and Society, vol. 2012, Article ID 406821, 17 pages, 2012.

[32] B. D. Iričanin and S. Stević, "Some systems of nonlinear difference equations of higher order with periodic solutions," Dynamics of Continuous, Discrete \& Impulsive Systems A, vol. 13, no. 3-4, pp. 499-507, 2006.

[33] A. Gelisken, C. Cinar, and I. Yalcinkaya, "On a max-type difference equation," Advances in Difference Equations, vol. 2010, Article ID 584890, 6 pages, 2010.

[34] C. Wang, S. Wang, L. Li, and Q. Shi, "Asymptotic behavior of equilibrium point for a class of nonlinear difference equation," Advances in Difference Equations, vol. 2009, Article ID 214309, 8 pages, 2009.

[35] C.-Y. Wang, S. Wang, Z.-w. Wang, F. Gong, and R.-f. Wang, "Asymptotic stability for a class of nonlinear difference equations," Discrete Dynamics in Nature and Society. An International Multidisciplinary Research and Review Journal, vol. 2010, Article ID 791610, 10 pages, 2010.

[36] X. Zhang, L. Liu, Y. Wu, and Y. Lu, "The iterative solutions of nonlinear fractional differential equations," Applied Mathematics and Computation, vol. 219, no. 9, pp. 4680-4691, 2013.

[37] A. S. Kurbanli, "On the behavior of solutions of the system of rational difference equations: $x_{n+1}=x_{n-1} /\left(y_{n} x_{n-1}-1\right)$, $x_{n+1}=y_{n-1} /\left(x_{n} y_{n-1}-1\right)$ and $z_{n+1}=z_{n-1} /\left(y_{n} z_{n-1}-1\right), "$ Discrete Dynamics in Nature and Society. An International Multidisciplinary Research and Review Journal, vol. 2011, Article ID 932362, 12 pages, 2011.

[38] K. Liu, Z. Zhao, X. Li, and P. Li, "More on three-dimensional systems of rational difference equations," Discrete Dynamics in Nature and Society, vol. 2011, Article ID 178483, 9 pages, 2011.

[39] S. Stević, "On a system of difference equations," Applied Mathematics and Computation, vol. 218, no. 7, pp. 3372-3378, 2011. 


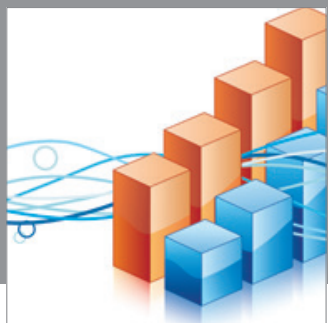

Advances in

Operations Research

mansans

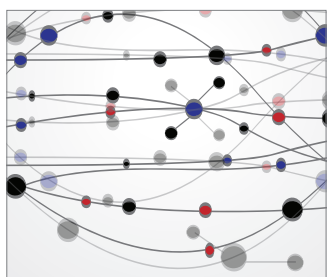

The Scientific World Journal
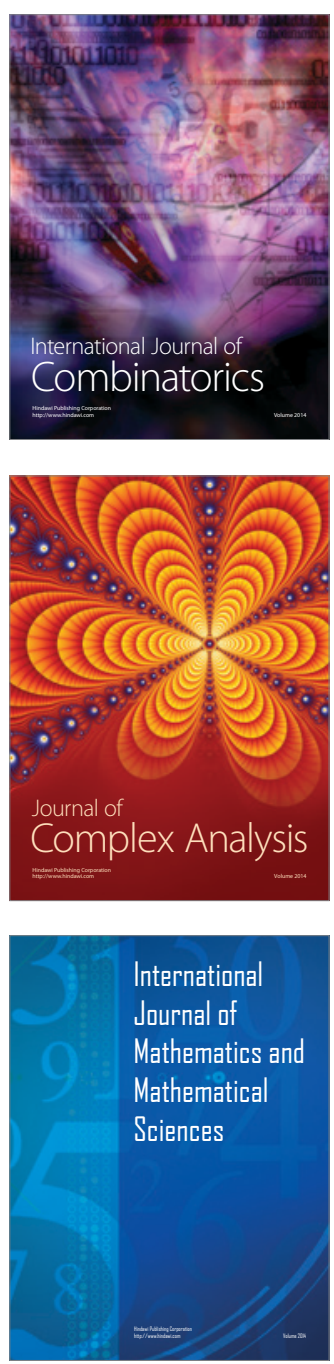


Submit your manuscripts at http://www.hindawi.com
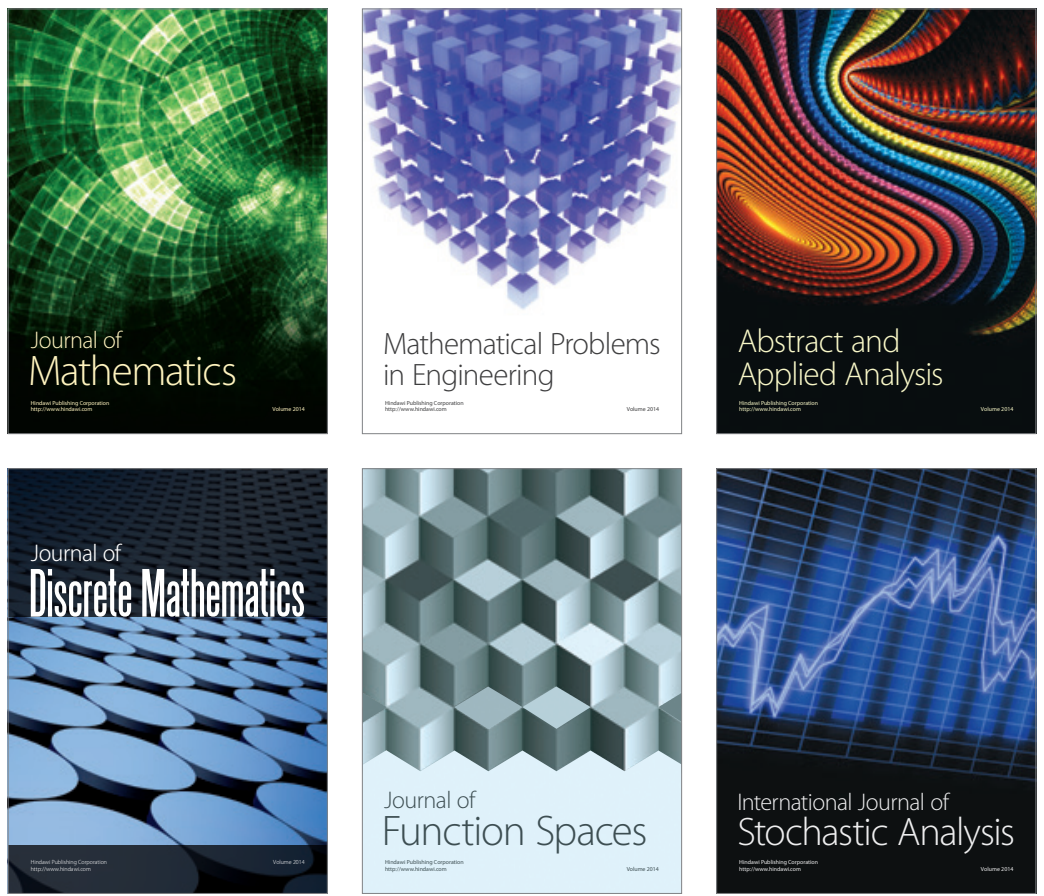

Journal of

Function Spaces




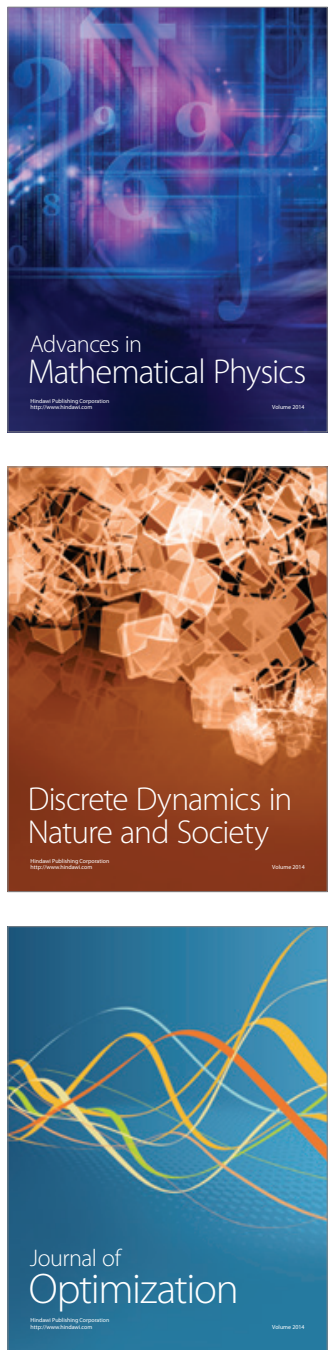